Copyright (C) 2021 University of Bucharest Printed in Romania. All rights reserved

ISSN print: $1224-5984$

ISSN online: $2248-3942$
Rom Biotechnol Lett. 2021; 26(6): 3110-3113 doi: $10.25083 / \mathrm{rbl} / 26.6 / 3110-3113$

Received for publication, September, 12, 2021

Accepted, November, 15, 2021

Original paper

\title{
Incidental Meckel's diverticula low grade neuroendocrine tumor coexisting with synchronous sigmoidian and perianal adenocarcinoma
}

\author{
SORIN PAUN ${ }^{1,2}$, IOAN TANASE ${ }^{1,2}$, BOGDAN STOICA ${ }^{1,2}$, CEZAR CIUBOTARU $^{2}$, \\ DIANA PAUN ${ }^{1}$
}

${ }^{1}$ University of Medicine and Pharmacy Carol Davila, Bucharest, Romania

${ }^{2}$ General Surgery Department, Emergency Hospital of Bucharest, Romania

\begin{abstract}
Although rarely, due to the high variability of the possible tissues encountered in it's wall, the Meckel's diverticula can represent a site for tumor development in $0,5-3,2 \%$ cases. Data in the literature shows that the most common malignancy at this level is represented by neuroendocrine tumors (NET) the observed incidence of neurendocrine tumors and other primary neoplasia, varies between $12-55 \%$. Moreover, the gastro-intestinal tract was the second primary tumor bearer encountered.
\end{abstract}

Keywords neuroendocrine tumor, Meckel, synchronous neoplasm

To cite this article: PAUN S, TANASE I, STOICA B, CIUBOTARU C, PAUN D. Incidental Meckel's diverticula low grade neuroendocrine tumor coexisting with synchronous sigmoidian and perianal adenocarcinoma. Rom Biotechnol Lett. 2021; 26(6): 3110-3113. DOI: 10.25083/rbl/26.6/ 3110-3113.

*Corresponding author: IOAN TANASE, University of Medicine and Pharmacy Carol Davila Bucharest,General Surgery Department, Emergency Hospital of Bucharest, Romania, No 8 Floreasca Street, Sect 1, 014461, Bucharest, Romania, Phone: +40744338049 .

E-mail: ioantanase@gmail.com 


\section{Introduction}

We present a patient admitted in the General Surgery Clinic of Bucharest Emergency Hospital with unspcecific abdominal symptomatology and that diagnosed with synchronous sigmoidian and perianal adenocarcinoma and a neuroendocrine tumor localised in the Meckel's diverticula.

\section{Background}

The presence of a Meckel's diverticula is the consequence of a failure in the closing of the vitelline duct during the $8^{\text {th }}$ week of intrauterine development and it represents the most common gastrointestinal malformation affecting aproximately $2 \%$ of the population[1]. Although rarely, due to the high variability of the possible tissues encountered in it's wall (gastric mucosa, pancreatic tissue, Brunner glands, colonic mucosa[2]), the Meckel's diverticula can represent a site for tumor development in $0,5-3,2 \%$ cases[2]. Data in the literature shows that the most common malignancy at this level is represented by neuroendocrine tumors (NET) $(76.5 \%$ of cases), followed by adenocarcinoma (11.4\%), GIST/ leiomyosarcoma and sarcoma (10.8\%), and lymphoma (1.3\%)[3]. NET's originating in the Meckel's diverticula are most often accidental findings, more than half of the patients being asymptomatic[4]. When present, symptoms are generally unspecific and include: abdominal pain, diarrhea, weight loss, nausea, and vomiting or they are correlated to the substances secreted by the tumoral tissue[4], [5]; however data in the literature shows that $24 \%$ of the patients had evidence of metastatic disease at the diagnostic moment[6], [7], a similar rate of dissemination when compared with the general metastasis incidence of the neuroendocrine tumors $-22 \%[8]$. Early diagnosis is more likely in the presence of hipersecretion expressed more often as a paraneoplasic or carcinoid syndrome. According to the World Health Organisation gastrointestinal neuroendocrine tumors are classified as grade 1 , grade 2 and grade 3 in terms of mitosis rate and Ki67 index, while neuroendocrine carcinomas (actively proliferating G3 tumors) are classified in small cell neuroendocrine carcinoma and large cell neuroendocrine carcinoma[9]. Cancer-specific survival of patients with grade 1, localized NETs is excellent, with approximately $97 \%$ survival at 5 years follow-up. Surprisingly, age appears to be associated with a worse prognosis independent of NET stage even in patients with lower grade disease, these having worse median overall survival compared to younger subjects[10].

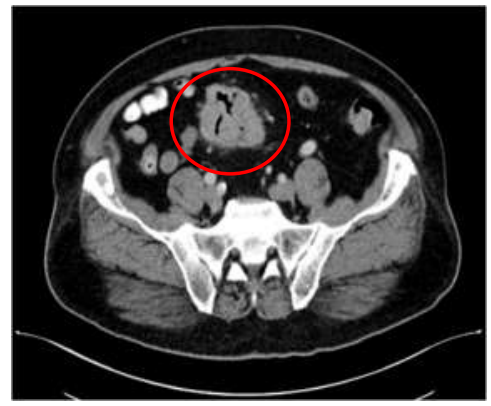

Figure 1. Sigmoidian stenosing tumor CT aspect
The majority of neuroendocrine tumors are sporadic, literature reviews suggest a possible association with Multiple Endocrine Neoplasia Syndrome I (MEN I) and in some cases familial clustering[11]. Once medical curiosities case reports of these particular situation have increased lately as observed by several authors in systematic literature reviews[8]. According to this data, the observed incidence of neuroendocrine tumors and synchronous (SPM) or metachronous primary neoplasia, varies between $12-55 \%[10]-[12]$ even in patients without genetic aggregation [8], [13]. Above all, the gastro-intestinal tract was the secondary primary tumor bearer in most of the cases[8]. A recent systematic literature review that analysed the presence of gastro-intestinal localised neuroendocrine tumors and a secondary primary malignancy found 76 cases [8]. The stomach and the large intestine are the predilect viscera for the SPM according to the literature. Adenocarcinoma accounted for almost the half of overall SPM, and $97.6 \%$ gastrointestinal neoplasia[8].

Although coexistence of colon adenocarcinoma and neuroendocrine tumor arising in a Meckel's diverticula have been previously reported[14], this situations represent undoubtedly a rarity; however coexistence of synchronous colorectal adenoacarcinoma and NET in Meckel's diverticula has never been reported in the literature from our knowledge.

\section{Case presentation}

A 65-year-old patient, known with hypertension, ischemic heart disease, acute myocardial infarction 16 years prior admittance, presented to our department for diffuse abdominal pain, intestinal transit disorders with alternating constipation with diarrhea and fatigue. The clinical examination revealed moderate distended abdomen with discrete pain at profound palpation in the left iliac fossa; at perineal level, in right posterior quadrant, lateral to the anal orifice, an $8 / 6 \mathrm{~cm}$ relatively well delimited, solid tumor, whitish, with central necrosis, and fistula with puss discharge. The tumor was adjacent to the right lateral wall of the anal canal; another tumor with same characteristics, but with smaller dimensions (about $1 \mathrm{~cm}$ diameter) in the left anterior quadrant

Blood tests showed mild leukocytosis $(12300 / \mathrm{mmc})$, mild thrombocytosis $(358000 / \mathrm{mmc})$, and hyperglycemia (259 $\mathrm{mg} / \mathrm{dl})$; increased CEA $(5.8 \mathrm{ng} / \mathrm{ml})$. No particularities at the abdominal ultrasound and chest and abdominal X-Rays. Colonoscopy reveals an ulcerated, stenotic tumor, $(75 \%$ of the colonic lumen), with diffuse bleeding at $25 \mathrm{~cm}$ from the anal verge; multiple in the descending and ascending colon.

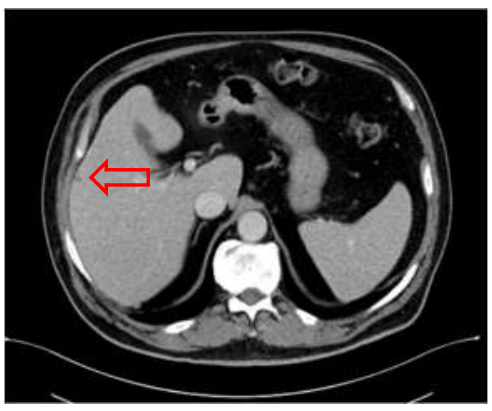

Figure 2. Hepatic lesion CT aspect 
Contrast thoraco-abdomino-pelvic CT scan, showed stenotic sigmoidian tumor with peritumoral and mesenteric vessels microadenopathies; hepatic tumor in the fifth with non-specific appearance with no signs of pulonary metastasis.

The patient underwent surgery through median incision, and a $10 / 8 \mathrm{~cm}$, stenotic, sigmoidian tumor, with invasion of the colon serosa and enlarged pericolonic lymph nodes were found; also an $1 \mathrm{~cm}$ hepatic tumor situated in the VII segment and a Meckel's diverticulum without inflamation were found. Wedge hepatic resection; sigmoidectomy with manual T-T colo-colo-anastomosis; diverticulectomy and ileostomy were performed; After patient repositioning ablation of perianal tumors that are firmly adherent to the right wall of the anal canal was performed.

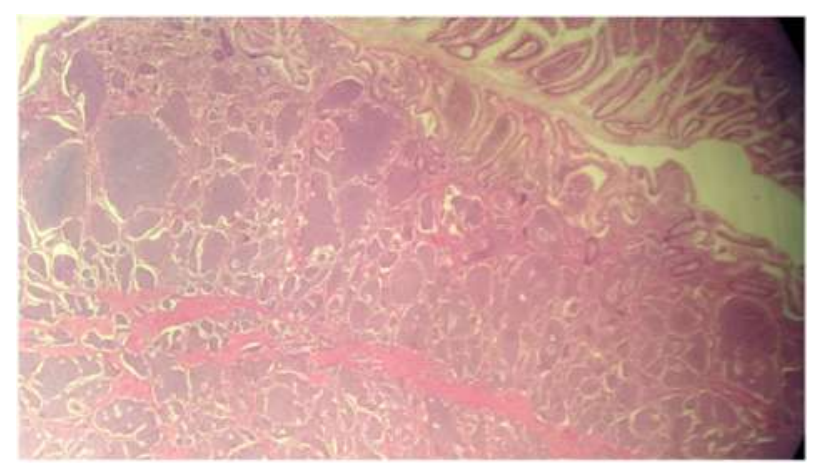

Figure 3. HE 100x Neuroendocrine tumor

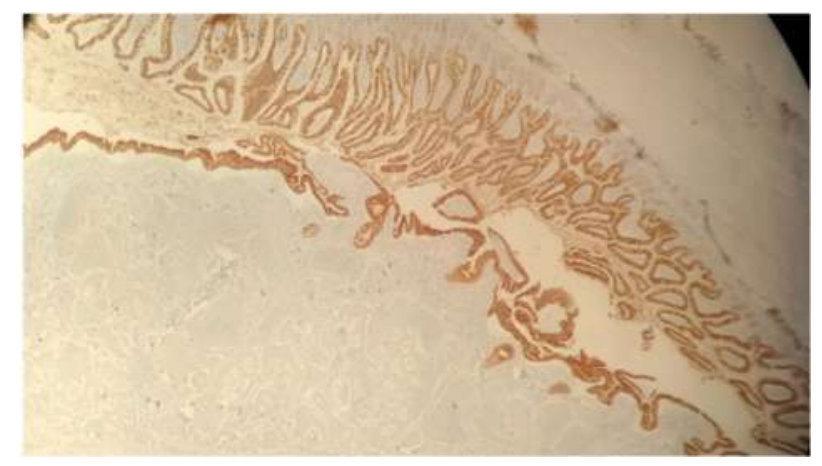

Figure 5. AE1/AE3

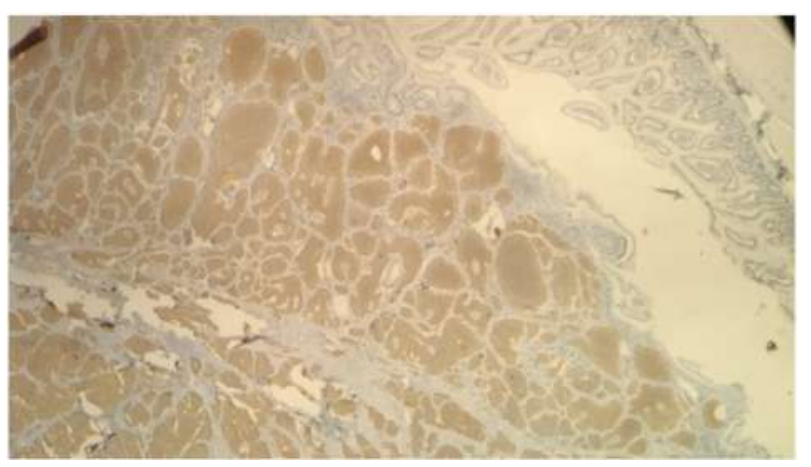

Figure 7. Synapthophisin
The patient's hospitalisation was uneventfull, with favorable evolution of the perineal remnant cavity and was discharged 18 days after the surgery.The histopathological result showed conventional colonic adenocarcinoma pT3N0, G2, both in the sigmoid colon and in the perianal region; Meckel diverticulum with grade I, localised neuroendocrine tumor $(1,5 \mathrm{~cm})$, developed in the mucosa, penetrating the scubmucosa and muscularis propria, without serosa involvment and no mucosal ulceration. Imunohistochimy tests shows: synapthophisin and chromogranin positive in tumor cells. Ki67 positive in less than $1 \%$ of tumor cells. DOG, CK7, CK20 negative in tumor cells. Postoperative tests showed 5-Hydroxyindolylacetic acid (5-HIA) = $2,76 \mathrm{mg} / 24 \mathrm{~h}$, serotonine serum levels $=132,75 \mu \mathrm{g} / \mathrm{L}$, and plsmatic Chromogranin-A = 34,48ng.mL

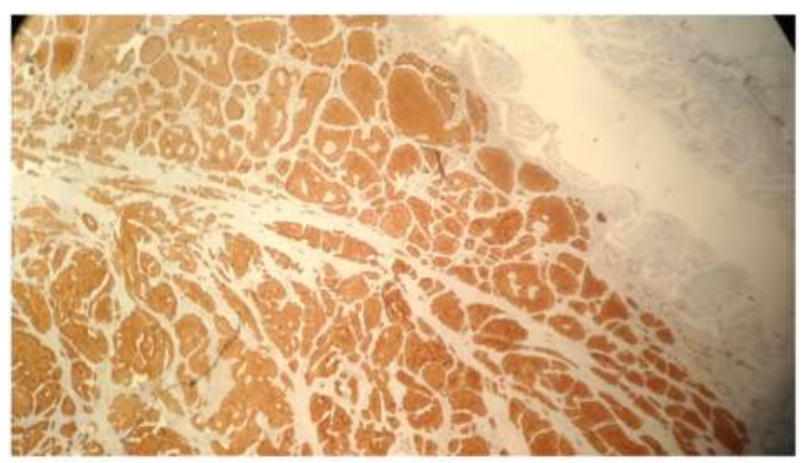

Figure 4. Chromogranin 100x

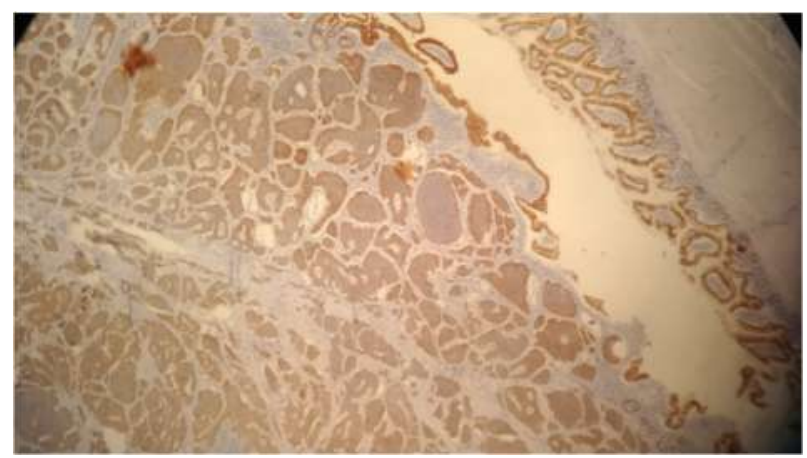

Figure 6. CK20

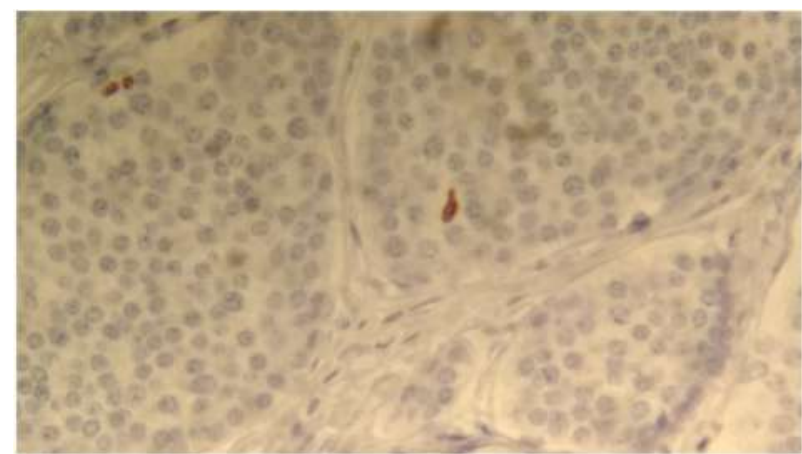

Figure 8. Ki67 
Liver tissue with preserved structure, showing small tumoral area, with uniform, medium-sized cells, with nucleolate nuclei, with IHC showing: Ki67 positive in 5\% of tumor cells that showed inflammatory aspects, CK7, CK20, synapthophisin, chromogranin, DOG, AE1/AE3 were found positive in rare hepatocytes and bile ducts; OCH1E5 positive in hepatocytes- appearrence of regeneratie liver nodule.

The patient is monitorized oncologically, but without adjuvant treatment. The patient is admitted to our service at 3 months postoperatively for stoma reintegration, without clinical and paraclinical signs of colonic or perineal recurrence.

Contrast abdominal MRI, at 3 months postoperatively, showed in the fifth liver segment a hyperplastic regenerative node, that requires imaging monitoring.

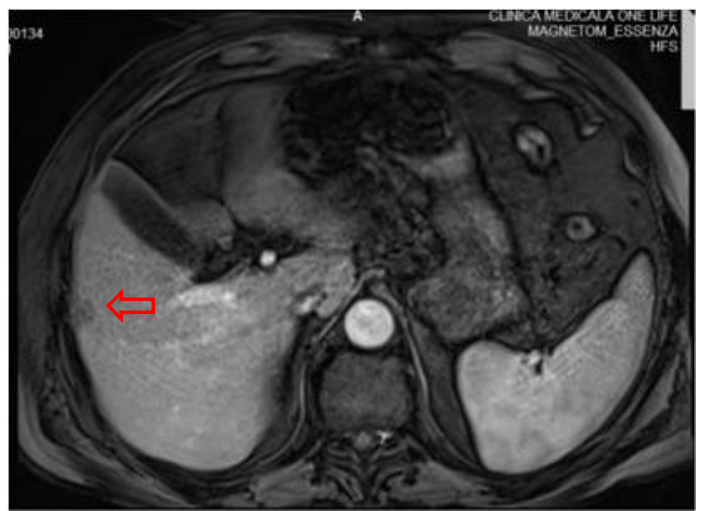

Figure 9. 3 months postoperative control MRI T1

\section{Conclusion}

The presence of multiple synchronous neoplasms in a patient with a neuroendocrine tumor back up the theory sustaining the carcinogenic properties of certain type of NET's or the personal predisposition in developing malignancies. The presence of multiple malignancies should not be looked upon as a rarity but arouse further research in order to identify a possible NET as an underlining cause.

\section{References}

1 E. K. Yahchouchy, A. F. Marano, J. C. F. Etienne, and A. L. Fingerhut, "Meckel's diverticulum," J. Am. Coll. Surg., vol. 192, no. 5, pp. 658-662, 2001, doi: 10.1016/S1072-7515(01)00817-1.

2 M. Yamaguchi, S. Takeuchi, and S. Awazu, "Meckel's diverticulum. Investigation of 600 patients in Japanese literature," Am. J. Surg., vol. 136, no. 2, pp. 247-249, 1978, doi: 10.1016/0002-9610(78)90238-6.

3 P. Thirunavukarasu et al., "Meckel's diverticulum-A high-risk region for malignancy in the ileum: Insights from a population-based epidemiological study and implications in surgical management," Ann. Surg., vol. 253, no. 2, pp. 223-230, Feb. 2011, doi: 10.1097/SLA.0b013e3181 ef488d.

4 I. M. Modlin, M. D. Shapiro, and M. Kidd, "An analysis of rare carcinoid tumors: Clarifying these clinical conundrums," World J. Surg., vol. 29, no. 1, pp. 92-101, Jan. 2005, doi: 10.1007/s00268-0047443-z.

5 D. L. Paun et al., "Cushing syndrome secondary to ectopic adrenocorticotropic hormone secretion from a Meckel diverticulum neuroendocrine tumor: Case report," BMC Endocr. Disord., vol. 15, no. 1, pp. 16, 2015, doi: 10.1186/s12902-015-0070-x.

6 C. Nies, A. Zielke, C. Hasse, J. Rüschoff, and M. Rothmund, "Carcinoid tumors of Meckel's diverticula - Report of two cases and review of the literature," Diseases of the Colon \& Rectum, vol. 35, no. 6. Springer-Verlag, pp. 589-596, Jun. 1992, doi: 10.1007/BF02050541.

7 D. J. Anderson, "Carcinoid tumor in Meckel's diverticulum: Laparoscopic treatment and review of the literature," Journal of the American Osteopathic Association, vol. 100, no. 7. American Osteopathic Association, pp. 432-434, Jul. 01, 2000, doi: 10.7556/jaoa.2000.100.7.432.

8 R. Parra-Medina, P. Moreno-Lucero, J. JimenezMoreno, A. M. Parra-Morales, and A. Romero-Rojas, "Neuroendocrine neoplasms of gastrointestinal tract and secondary primary synchronous tumors: A systematic review of case reports. Casualty or causality?," PLoS One, vol. 14, no. 5, pp. 1-16, 2019, doi: 10.1371/journal.pone.0216647.

9 G. Rindi, R. Arnold, and F. Bosman, "Nomenclature and classification of neuroendocrine neoplasms of the digestive system," WHO Classif. Tumours Dig. Syst., pp. 13-14, Jan. 2010.

10 P. E. Sackstein, D. S. O’Neil, A. I. Neugut, J. Chabot, and T. Fojo, "Epidemiologic trends in neuroendocrine tumors: An examination of incidence rates and survival of specific patient subgroups over the past 20 years," Semin. Oncol., vol. 45, no. 4, pp. 249-258, 2018, doi: 10.1053/j.seminoncol.2018.07.001.

11 B. G. Taal and O. Visser, "Epidemiology of Neuroendocrine Tumours," Neuroendocrinology, vol. 80, no. 1, pp. 3-7, 2004, doi: 10.1159/000080731.

12 I. M. Modlin, K. D. Lye, and M. Kidd, "A 5-decade analysis of 13,715 carcinoid tumors," Cancer, vol. 97, no. 4, pp. 934-959, Feb. 2003, doi: 10.1002/cncr.11105.

13 A. K. Clift et al., "Incidence of Second Primary Malignancies in Patients with Neuroendocrine Tumours," Neuroendocrinology, vol. 102, no. 1-2, pp. 26-32, Dec. 2015, doi: 10.1159/000381716.

14 D. Katalinic, F. Santek, A. Juretic, D. Skegro, and S. Plestina, "Gastroenteropancreatic neuroendocrine tumour arising in Meckel's diverticulum coexisting with colon adenocarcinoma," World J. Surg. Oncol., vol. 12, no. 1, pp. 1-7, 2014, doi: 10.1186/1477-781912-358. 